\title{
GICS, a new concept to enhance in-flight safety and security of commercial aviation
}

\author{
Marc Lesturgie ${ }^{1,1}$, Hian Lim Chan, and Sylvain Azarian \\ ${ }^{1}$ ONERA (The French Aerospace Lab), Chemin de la Hunière, 91123 Palaiseau CEDEX, France
}

\begin{abstract}
This paper presents a new concept of aircraft tracking based on a LEO constellation of small satellites, each of them carrying receiving and transmitting devices in order to check the integrity of the ADS-B (Automatic Dependent Surveillance - Broadcast) positioning information and proceed with a non-cooperative independent localization of the aircraft, if a flight anomaly comes out. To do so, on side of the conventional ADS-B processing, authors propose to add a multilateration process and two active radar modes, able to perform a finer and reliable localization of the aircraft, as well as a first information on the nature of the flight anomaly. The paper deals with a preliminary system analysis, although underpinned by some illustrations of existing sensor technologies, already or nearly operational in space. The concept has been patented [1].
\end{abstract}

Keywords- ADS-B, SAR, Radar, ATC, multilateration

\section{Introduction and context}

The aviation industry has always lived with the fact that aircrafts are devilishly difficult to track over large bodies of water since the dawn of transoceanic flights in 1919. Figure 1 gives a sensing of the density of cross-continental flights over oceans. They are also aware that most of the current aircraft tracking technologies will have problems if an external action comes into play to manipulate them. Triggered by the various aviation disasters like AF447, MH374, MH17, QZ8501, the ICAO is pushing for any solution in a bid to show that they are acting.

European airlines are already required by the European Aviation Safety Agency (EASA) to track positions of their aircrafts at least once every 15 minutes. The 15 -minute reporting requirement was brought in by ICAO as a result of the losses of Air France Flight 447 and Malaysia Airlines Flight MH370. A new policy (since November 2016) covers the tracking of commercial aircrafts at 15 minute update rate and with a position report accuracy of 1 nautical mile, and with the ability to increase the fluency of the tracks to 1 minute if the aircraft shows sign of getting into danger with deviation from their normal positions or tracks.

This paper proposed a new concept based on a constellation of LEO satellites able to check the integrity of the positioning data and to localize in a non-cooperative manner any commercial aircraft victim of a failure arising from an intentional or non-intentional nature.

${ }^{1}$ Corresponding author: marc.lesturgie@onera.fr 
The concept, illustrated by the figure 2 is therefore able to enhance both the in-flight safety and security levels.

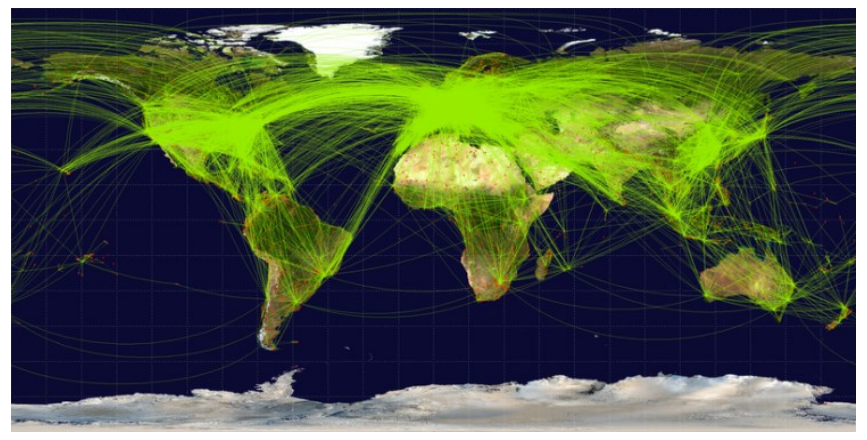

Fig. 1. Map of air traffic [from https://www.northpointaviation.com/]

\section{Today's solutions and limitations}

Aircrafts detection and tracking are achieved by primary and secondary air traffic surveillance sensors over land, as well as organic tracking / reporting devices (carried by aircrafts) for flights over oceanic and remote areas. The latter solutions usually require ground-based and space-based supporting infrastructures.

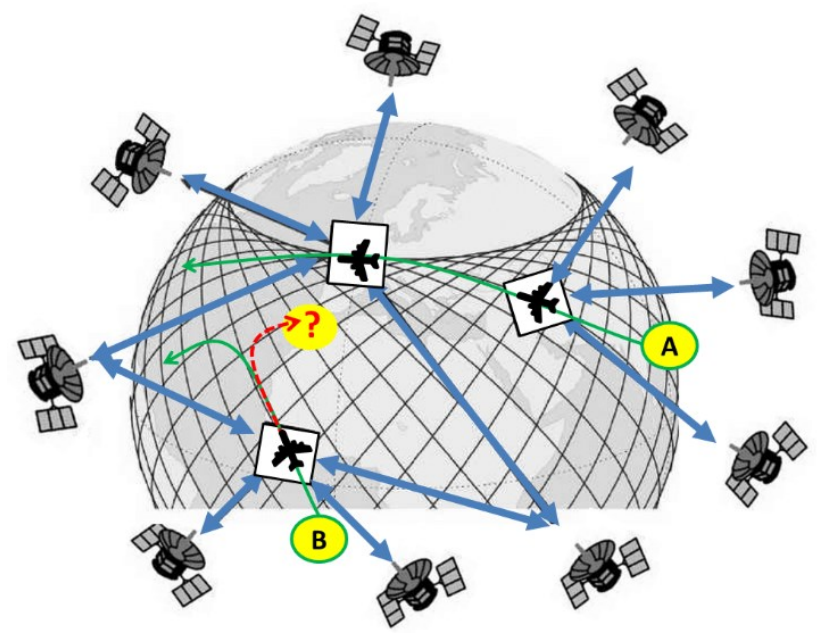

Fig. 2. Illustration of the concept; track $A$ is a nominal route while B is presenting an anomaly (spoofing of ADS-B), to be detected on time by the constellation. Green lines represent the ADS-B trajectory.

\subsection{Air Traffic Surveillance over land areas}

Most primary radars used for long range surveillance Air Traffic Control (ATC) are operating in the L or S-Bands. Other than for surveillance purpose, these primary radars are used also for airspace infringement protection and airport surface surveillance. L-Band electromagnetic waves do not follow the curvature of the earth; at low altitude the range is limited because the aircraft are below the horizon. At higher altitudes the range is up to about $250 \mathrm{NM}$, but only when the radar is operated at very high power, which is costly both in design and electricity bill. In many cases, it is not needed to operate at long range. Radar is also shielded by terrain; in mountainous areas it is difficult to cover the valleys. In Europe 
and in the US, high altitudes are well covered by primary radar, however at low altitude there are many coverage gaps.

\subsection{Air Traffic Surveillance Over Oceans and Remote Areas}

Most airspace above seas and oceans are not covered by ATC primary radars. Today in oceanic airspace, pilots report their position and other information at certain waypoints using high-frequency (HF) radio reports or digitally via data link over HF links or satellite networks. But these reports can be as sparse as $60-90$ minutes depending on the geographical locations.

\subsection{ADS-B Tracking of commercial aircraft}

Transponders carried by the aircrafts provide position data sent from the aircrafts to the receive terminals on the ground, on air or space. About $80 \%$ of the world's landmass is already covered with ADS-B (about 6,200 receivers), expanding at the rate of 50 new receivers installed every week, and is the largest among a growing number of privately owned aircraft surveillance networks. The receivers, many provided at no cost by company like FLIGHTRADAR24, are placed at homes and businesses of ardent supporters. The ADS-B position and identification outputs from the aircrafts flying over land, are captured at 1 second update rate. About half of the receivers today are also tracking aircraft carrying legacy "Mode $\mathrm{S}$ " transponders using a form of triangulation called multilateration.

For over ocean flights, the aircraft ADS-B data is sent to a ground land-based terminal via Inmarsat and Iridium satellite constellations (such satellite tracking technologies are expensive within the airline industry) or through ARINE's ground-based HFDL network.

FLIGHTRADAR24 has been experimenting ADS-B receivers attached to buoys or autonomous boat, a relatively low-cost solution [2]. Interest is of course to have buoys in captive locations throughout the oceans or left to slowly free-float on the currents between continents.

AIREON has been working (from 2015) on an ADS-B receiver on-board of a communications satellites. AIREON's technology does not require any change to airliners operating around the world. Their Space-based ADS-B solution [3] provides full, continuous, global air traffic surveillance, and insuring safety in difficult regions i.e. the oceans, Polar Regions, mountainous regions, jungles, deserts. There are a total of 66 Iridium NEXT satellites, with significant overlap and redundancy.

\subsection{Limitations of the current solutions}

ADS-B including space-borne receivers contributes to a major evolution of the safety system for the tracking of commercial aircrafts. However, the solution - as the genuine ADS-B system relies on a transmitting transponder on board of the aircraft, and a proper cooperative decoding of the signals. In case of failure of the transponder unit or in case of an intentional spoofing of the transponder, the cooperative decoding becomes impossible, or even worse, corrupted, without the controllers and pilots being aware.

The various assumptions or reports[4], following the disappearance of the MH370 flight are all mentioning a failure of ADS-B transponding signals, and a suspicion of intentional cut of the transponder [8], at a right time which may corresponds to the transition between two ATC areas.

\section{A global integrity check system (GICS)}




\subsection{Re-introducing non-cooperative tracking}

Primary radar tracking has left room to secondary radar and cooperative tracking is also assessed to be more efficient than non-cooperative tracking. The idea of course is not to reintroduce primary radars for replacement of secondary radars but to compliment the current system with a non-cooperative tracking in order to perform:

1. An integrity check that delivers a tamperproof

2. In case (and only in case) of anomaly, to perform the non-cooperative tracking and localization.

One way to check the integrity of a position given by the normal decoding of the transponder (ADS-B) signals is to obtain a second (and independent) measurement of the position of the aircraft. ADS-B relies on a selective protocol, since each aircraft is being associated to its ICAO code. Therefore it is possible to extract the transponder signals associated to the same ICAO code from one or several receiver. Considering 3 receivers from space (figure 3), at different positions, we can proceed with multilateration (either in time of Doppler domain) to derive a second measure of the aircraft position.

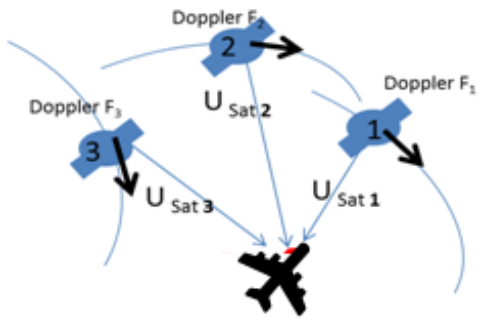

Fig. 3. The multilateration principle

The concept named GICS [1] (for Global Integrity Check System) covers the following tasks:

3. Check the position integrity of any commercial aircraft from comparing the ADS-B position and the position derived by multilateration applied to selective transponder signals $^{2}$,

4. Detect any ATM anomaly, 7/24 with a non-cooperative strategy,

5. Proceed with the localisation of the anomaly (loss, hijacking, crash) within a radius of $30 \mathrm{~km}$, (including any kind of drift)

All sensors mentioned below are spaceborne. The satellites have data links (to ground station), and equipments for time and frequency synchronization which are not described in this paper. To perform the whole mission, the concept is split into three sequential steps, each step being associated to a specific operating mode.

\section{Step 1: multilateration / semi cooperative mode}

Techniques based on frequency difference of arrival (FDOA) analysis are used to check the integrity of the position delivered by ADS-B signal. However, for reasons of complexity (computational load), instead of computing the $\mathrm{x}, \mathrm{y}, \mathrm{z}$ position of the aircraft we compute an integrity test by comparing the estimated and measured Doppler values.

As depicted by the figure 4, ADS-B signals are decoded according to a normal process, providing the localization and velocity of the aircraft (outcome of DEMUX). These data are

${ }^{2}$ In case the transponder is switched off, the latest position of the aircraft derived from step 1 is used. We assume that the transponder is working nominally after aircraft take-off. 
used to model the Doppler values that should be observed by each of the 3 satellites involved in the so-called multilateration process. On the other hand, analog signals received from the ADS-B receiving chain are processed to derive the measured (estimated) Doppler values. A Matching unit is implemented to compare the measured and modeled Doppler frequencies and decide whether the trajectory is normal or abnormal. The process is applied across time, integrating several snapshots in order to reduce the false alarm / false decision at the output of the Matching unit. An important aspect will be the tuning of the decision threshold, which is not discussed in this paper.

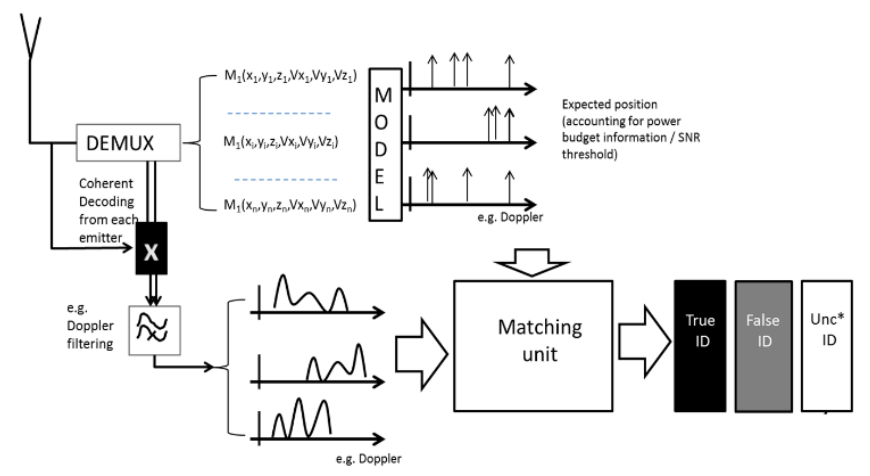

Fig. 4. The decision chain, based on comparison of measured and modeled Doppler frequencies

\section{Step 2: Track the anomaly (if any) with a primary radar}

Upon detection of the anomaly we switch on the active radar. The active radar is either carried by one of the satellites used at step 1 or by another satellite getting closer, hence providing optimal conditions of detection in terms of signal to noise ratio. Under this step, the beam of this active radar is steered to the position of the anomaly, known from step 1 . The selection of the optimal satellite w.r.t. the detection performances, as explained before may induce an additional time (the handover time) during which the position of the anomaly may suffer from a drift (in flight, the drift is a function of the aircraft velocity). Considering this effect, the radar must provide a sufficient wide area of coverage, to anticipate the spread of the position due to this additional drift.

Different radar technologies are potentially good candidates to support this non cooperative mode / primary detection.

Among them, we can list:

- The active UHF radar, efficient to detect and track commercial aircraft at long distances

- Passive radar technology, a technology that emerged in the 90's and is using transmitters either carried by satellite, aircrafts (not only the one of interest) or ground based transmitters (radio, TV, radar..)

\section{Step 3: Refine the localization, image and classify}

The step 3 aims to:

- Trigger / cue a microwave radar operating with an imaging mode (SAR or ISAR)

- Provide localisation of the anomaly within an area of a few tens of kilometres

- Classify the event / disaster (e.g. hijacking, crash, spread of debris) 
Table 1 summarizes the general characteristics of the sensors

\begin{tabular}{|l|l|l|}
\hline Step & Function & Sensor technology \\
\hline 1 & $\begin{array}{l}\text { - Decode ADS-B from space } \\
\text { - Semi-Cooperative mode, with multilateration } \\
\text { - integrity check of the ADS-B position } \\
\text { - detect anomaly (switch of transponder, deviation,...) } \\
\text { - cueing step / mode 2 }\end{array}$ & $\begin{array}{l}\text { - L band receivers } \\
\text { - Multilateration }\end{array}$ \\
\hline 2 & $\begin{array}{l}\text { Non-cooperative detection mode, able to cover a } \\
\text { wide area, providing a CEP around 10km }\end{array}$ & $\begin{array}{l}\text { - Active radar UHF band - passive } \\
\text { radar to compliment Radar }\end{array}$ \\
\hline 3 & $\begin{array}{l}\text { Imaging and fine localization mode, able to cover a } \\
\text { few 10km area, with a resolution a few meters }\end{array}$ & $\begin{array}{l}\text { X-band SAR/ISAR (active radar) } \\
\text { Compatible <100kg satellite }\end{array}$ \\
\hline
\end{tabular}

\subsection{Sequencing of the modes}

Each mode and sensor is also characterized by the following features (figure 5):

- The field of view (FoV)

- The footprint (FP) of the active modes (1) and (2), i.e. the focused beam area

- The accuracy of localisation (CEP)

The cueing strategy - in terms of time sequencing - is to consider that the FoV of the cueing mode is at least equal to the CEP of the previous mode to which we add a drift proportional to the handover time $\Delta_{\text {handover }}$ between two modes multiplied by the velocity of the aircraft Vac. Figure 5 illustrates the different modes, from step 1 on left to step 3 on right. For example, handover from Mode 1 (blue) to Mode 2 (green) requires

$$
\mathbf{F P}_{2}=\mathbf{C E P}_{1}+\Delta_{\text {handover. }} \mathrm{V}_{\mathrm{ac}}
$$

(a radius of 50 to $100 \mathrm{~km}$ for the FoV is able to tolerate a drift of the CEP equal to 20 to $50 \mathrm{~km})$

Similarly, transition from mode 2 (green) to Mode 3 (yellow) shall satisfy:

$$
\mathbf{F P}_{3}=\mathbf{C E P}_{2}+\Delta_{\text {handover. }} \mathbf{V}_{\text {ac }}
$$

The worst case corresponds to a handover time between 2 satellites and to the maximum radial speed of $300 \mathrm{~m} / \mathrm{s}$. If we assume a constellation with a uniform distribution of LEO satellites at $500 \mathrm{~km}$ altitude with an orbit inclination angle of $55^{\circ}$ to cover most of the global major aviation activities, we are able to derive the minimum number of satellites:

Table 2 : A first estimate of the minimum required number of satellites as a function of the size of the search and rescue area

\begin{tabular}{|c|c|}
\hline Radius of the area & Number of satellites \\
\hline $15 \mathrm{~km}$ & 98 \\
\hline $30 \mathrm{~km}$ & 49 \\
\hline $45 \mathrm{~km}$ & 33 \\
\hline $75 \mathrm{~km}$ & 20 \\
\hline
\end{tabular}

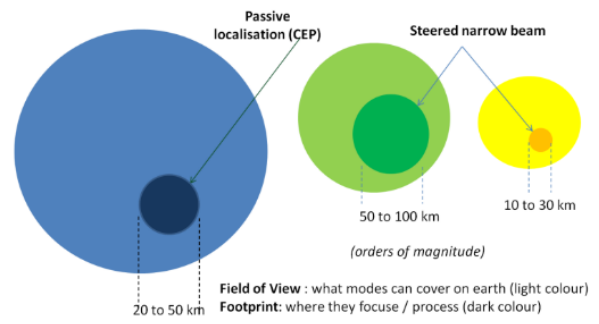

Fig. 5. Field of view and footprint of each mode 


\subsection{The search and rescue mode}

Radar imaging, unlike optical imaging, is weather tolerant and can image any object at long distance, even through clouds. Equatorial regions are, from this perspective highly interested in having spaceborne radar sensors, in addition to optical sensors. One interesting property of the radar is also its ability to process and classify objects from the Doppler signature. SAR (Synthetic Aperture Radar) is massively using Doppler processing to image land, sea / ocean surface. Any floating debris (of a reasonable size) can be detected using this technology, as far as the duration of the GICS mission is reasonable in regards to the flotation of debris for example.

Other radar techniques, derived from SAR and called ISAR (Inversed Synthetic Aperture Radar) are also able to image moving / flying objects under certain hypothesis. The objective of this paper is not to detail this technique, but to raise the fact that by combining several hypotheses, we would be able to classify, whether the anomaly is a spoofed situation, hijacking, a distress or a crash / impact producing debris (figure 6).

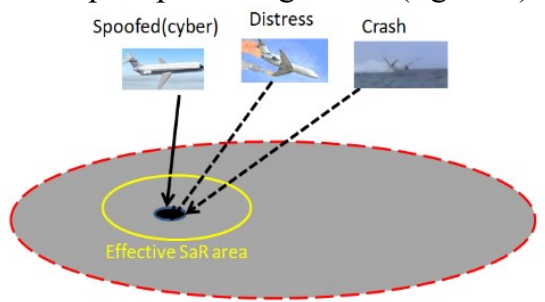

Fig. 6. Anomaly spoofed, hijacking, a distress or a crash / impact producing debris situation

\subsection{Sensor technology}

We assume a 100kg-class payload for each satellite unit and assume that each unit is carrying the 3 modes associated to the 3 aforementioned steps. However, only the passive mode 1 (step 1) is a permanent mode. Active modes (which are the most demanding in terms of energy supply) are only engaged in case of anomaly or in case of false alarm which we want to be as low as possible.

Figure 7 gives a schematic view, how to combine the 3 sensors on board of one satellite unit.

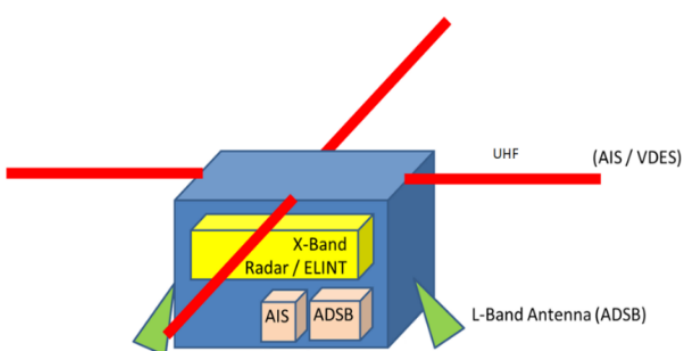

Fig. 7. Schematic view of the satellite payload

Among the technical challenges, one is currently to design the active X-band radar for imaging and classification purposes. The current state of art in terms of active compact and light weight SAR is illustrated by 2 projects:

- The ICEye project consists of a constellation of 18 satellites, each of them having a light weight below $100 \mathrm{~kg}$. 10 satellites should be launched in 2019. The SAR resolution can be as finer as 1 meter for the latest generation (ICEYE-X3), still with a weight not exceeding $100 \mathrm{~kg}$ [5]. 


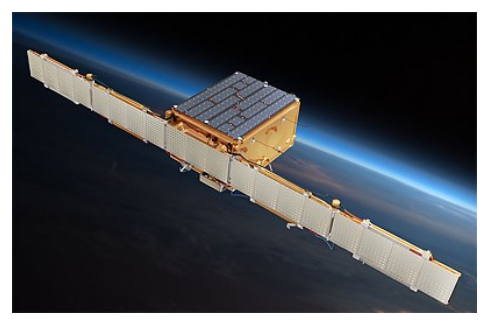

Fig. 8. The ICEYE-X1 satellite, from [5]

- JAXA and Keio University have developed a compact SAR of 100kg [6] equipped with a deployable plane antenna. A first satellite should be launched in 2019. The SAR resolution is around 3 meters which is sufficient for the GICS application.

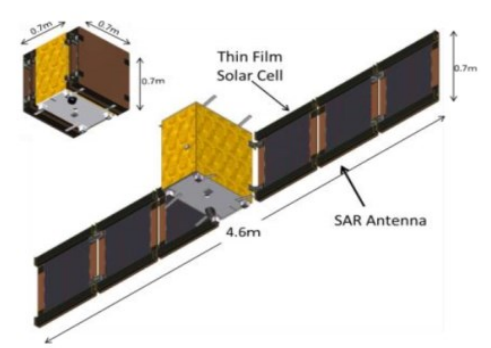

Fig. 9. A 100kg-class X-band compact SAR studied at JAXA [7]

Figure 10 extracted from a paper published by JAXA [7] displays the resolution of existing and future micro-Satellite (SAR mission), with a particular emphasis in the range of 3-10 meters resolution and a weight of $100 \mathrm{~kg}$. The recommended frequency band is the X-band, providing a good compromise between the antenna size and the power budget characteristics.

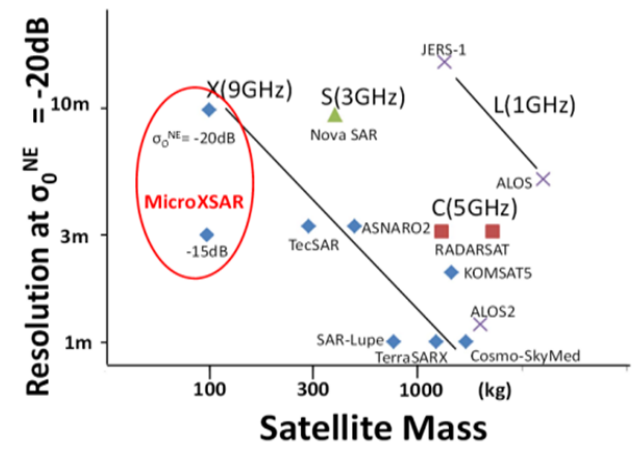

Fig. 10. Satellite mass versus SAR resolution, from JAXA [7]

\section{Conclusion}

The paper presents the basic design of LEO constellation satellites able to enhance the safety and security of commercial flights. The MH370 unexplained disappearance is still in the memory when engineers started looking at this concept. In the meantime, during the last 5 years new technologies made space much more affordable than 10 years ago. A 'new space 
era' has emerged, with mini or micro satellites concepts and a lot of small companies investing in this market.

GICS concept is made of cooperative and non-cooperative procedures to check the integrity of the aircraft position, 7/24 and on any localization of the globe. The preliminary study shows that with less than 50 satellites, we could reduce the size of the investigation down to $30 \mathrm{~km}$. Each satellite is equipped with a 3 -mode sensor, able to provide ADS-B decoding, multilateration, primary radar detection and imaging. Active radar requires a higher complexity, because of the antenna size, the power level and the quality (phase stability) of the transmitting signals.

The state of art for sensor technology is also encouraging. AEREON based on 66 Irridium satellites will soon become operational, providing ADS-B from space. Space-borne SAR using lightweight satellites (less than $100 \mathrm{~kg}$ ) is also real and some research teams are working on the optimization of the payload, for example to reduce the size of the antenna or use a foldable / deployable antenna much affordable in terms of room to reserve in the launcher. Upon the completion of the preliminary study, the main targeted application is to counter cyber-attacks and vulnerability resulting from the ADS-B fully cooperative strategy. Remote area, not covered by land-based equipments would benefit from this system. Beside countercyber-attacks, there are some other applications, like reducing the separation distances between commercial aircrafts, benefiting from this concept, especially in those areas where no ATC cooperation is established between nations.

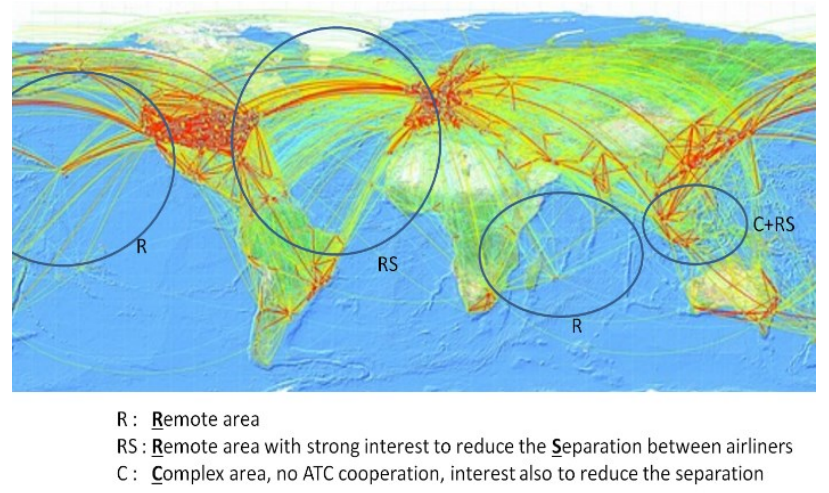

Fig. 11. Other applications of GICS, including the interest to reduce the separation distances between aircrafts

Figure 11 - Other applications of GICS, including the interest to reduce the separation distances between aircrafts

\section{References}

1. Patent PCT/SG2018/050125 Global integrity check system and associated method; https://patentscope.wipo.int/search/en/detail.jsf?docId=WO2018174822

2. https://www.flightradar24.com/blog/setting-sail-for-global-coverage-flightradar24-adsb-receivers-on-board-a-surface-ocean-robot/

3. AIREON

4. http://mh370.mot.gov.my/MH370SafetyInvestigationReport.pdf

5. https://www.iceye.com/resources/missions/iceye-x3

6. S. Shirasaka, 100kg-class X-band Synthetic Aperture Radar Satellite System for OnDemand Observation (International Radar conference, Toulon 2019)

7. http://www.isas.jaxa.jp/home/saito_hirobumi_lab/_src/sc1242/SAR.pdf

8. M. Delarche J.-M. Garot, Le détournement $\overline{d u} M \bar{H} 370$, (Librinova, ISBN 979-10-2620641-5) 